\title{
Filter Out High Frequency Noise in EEG Data Using The Method of Maximum Entropy
}

\author{
Chih-Yuan Tseng and HC Lee ${ }^{\dagger}$ \\ Department of Physics and ${ }^{\dagger}$ Graduate Institute of Systems Biology and Bioinformatics \\ Computational Biology Laboratory \\ National Central University, Chungli, Taiwan 320, ROC
}

\begin{abstract}
We propose a maximum entropy (ME) based approach to smooth noise not only in data but also to noise amplified by second order derivative calculation of the data especially for electroencephalography (EEG) studies. The approach includes two steps, applying method of ME to generate a family of filters and minimizing noise variance after applying these filters on data selects the preferred one within the family. We examine performance of the ME filter through frequency and noise variance analysis and compare it with other well known filters developed in the EEG studies. The results show the ME filters to outperform others. Although we only demonstrate a filter design especially for second order derivative of EEG data, these studies still shed an informatic approach of systematically designing a filter for specific purposes.
\end{abstract}

Keywords: EEG, Current source density, Non-recursive filter, Maximum entropy

PACS: $87.10+\mathrm{e} ; 87.57 . \mathrm{Gg}$

\section{INTRODUCTION}

Filtering out high frequency noise becomes extremely crucial in source localization problem of invasive EEG studies. Based on Freeman and Nicholson's works, source localization requires calculation of second order derivative of the EEG data $[1,2]$. Because the derivative amplifies especially high frequency noise, Freeman and Nicholson proposed to introduce low-pass non-recursive filters to smooth it. [2]. Suppose a set of discrete data, $\Phi(r)$ labeled by spatial position $\mathrm{r}$, which are distorted by noises $\varepsilon_{r}$ and these data are equally spaced sampled with interval $h$. The simplest non-recursive filter is then defined by simply averaging data at $\mathrm{r}$ and its neighboring data with specific set of weighting values $p_{l}$,

$$
\Phi_{s}(r)=\sum_{l=-z}^{z} p_{l} \Phi(r+l h)
$$

where $z$ is an integer and $2 z+1$ data points are considered. We will designate this $2 z+1-$ point spatial filter as a $S_{(2 z+1)}$ filter. The mean filter, in which $p_{l}$ equals to $1 /(2 z+1)$, is one filter widely used in many fields for data smoothing. Its applicability, however, is sometimes limited to data types [3].

Many methods have been developed to design low-pass filters in many fields. For example, there are adaptive beam-forming ([4] - [7]), wavelet de-noising [8], and entropic method ([9] - [14]) etc.. Yet no explicit discussions are given on smoothing noise in second order derivative of contaminated EEG data since Freeman and Nicholson's pioneering works. Freeman and Nicholson's approach will be briefly discussed in Sec. 2 . 
Two drawbacks, requirements of some empirical guidelines and low accuracy of second order derivative, in their approach will also be addressed.

Our goal is to provide a ME based approach to filter out high frequency noise especially in second order derivative of contaminated EEG data. This approach eliminates the two drawbacks in Freeman and Nicholson's approach and provides a systematic and robust method for smoothing noise. The ME approach and its application in the EEG studies will be presented in Sec. 3 and 4 respectively. We thereafter examine the proposed filter's performance and compare to some other filters such as the mean filter, Freeman and Nicholson's [2], and Rappelsberger's [15] filters through frequency and noise variance analysis in Sec. 5. A conclusion is given at last.

\section{SOURCE LOCALIZATION FROM THE INVASIVE EEG DATA}

In neurophysiology, electric field potentials are initiated during neural activity when ions flow across localized regions of cell membranes. These ionic flows establish a distribution of current sources and sinks in the extracellular space $[1,2,16]$. These field potentials can be measured in extracellular space discretely by the invasive EEG instrument, which usually has 16 to 32 detectors on its probe.

According to theory of electromagnetism, the current sources and sinks are associated with second order derivative of the field potentials. Freeman and Nicholson proposed the current source density (CSD) method for source localization [1,2]. The CSD computes second order derivative approximately through substituting specific smoothed $\Phi_{s}(r)$, Eq. (1), into $\mathrm{Ta}_{3}(r)=\sigma_{c} / h^{2}(\Phi(r-h)-2 \Phi(r)+\Phi(r+h))$, where three data are considered, $\sigma_{c}$ is conductivity, and $h$ is separating distance between two sensors. A final expression of an approximated second order derivative is given by

$$
D_{2}(r)=\sigma_{c} / h^{2} \sum_{m=-M}^{M} a_{m} \Phi(r+m h),
$$

from $2 \mathrm{M}+1$ datum with weightings $a_{ \pm M}=p_{z}, a_{ \pm M \mp 1}=p_{z-1}-2 p_{z}$, and $a_{ \pm M \mp k}=$ $p_{z-k}-2 p_{z-k+1}+p_{z-k+2}$, in which $k \geq 2$. The preferred smoothing function should be able to reduce high frequency noise and noise amplified by operation of the $\mathrm{Ta}_{3}(r)$. Freeman and Nicholson devised several smoothing functions as shown in Table. 1, denoted by FNSn ([2]), where Sn stands for n-point spatial smoothing. Notes that the $\mathrm{FNS}_{3}(2)$ is still used in Karwoski et al. [17] and Ulbert et al. [18]. These $p_{l}$ are derived from the least square method $[19,20]$. In addition, they also introduce some ad hoc rules to obtain 5 and 7-point filters. For example, the $\mathrm{FNS}_{5}$ is obtained by smoothing data twice using set of $(3 / 10,4 / 10,3 / 10)$. Later, several successors have designed other smoothing functions according to their needs such as the Rappelsberger's $\mathrm{RS}_{3}$ filter [15].

There are two drawbacks in Freeman and Nicholson's CSD method. The first is requirements of some ad hoc empirical guidelines for designing smoothing functions such as $\mathrm{FNS}_{3}(1)$ or $\mathrm{RS}_{3}$ shown in Table. 1. These empirical guidelines may limit the use of these smoothing functions to some specific EEG data. For example, Freeman and Nicholson's smoothing functions are suited for Anuran cerebellum studies [2]. The second is these smoothing functions are devised for second order derivative calculated via 
$\mathrm{Ta}_{3}(r)$, which is not an accurate calculation. In next section, we propose an informatic approach based on the ME method to eliminate these two drawbacks.

TABLE 1. Filters in EEG studies. The table lists five smoothing functions [2,15]. MF: mean filter, FN: Freeman and Nicholson, R: Rappelsberger.

\begin{tabular}{|l|cccc|}
\hline & $p_{0}$ & $p_{ \pm 1}$ & $p_{ \pm 2}$ & $p_{ \pm 3}$ \\
\hline $\mathrm{MFS}_{3}$ & 0.33 & 0.33 & 0 & 0 \\
$\mathrm{FNS}_{3}(1)$ & 0.5 & 0.25 & 0 & 0 \\
$\mathrm{FNS}_{3}(2)$ & 0.43 & 0.29 & 0 & 0 \\
$\mathrm{RS}_{3}$ & 0.54 & 0.23 & 0 & 0 \\
FNS5 & 0.34 & 0.24 & 0.09 & 0 \\
FNS7 & 0.26 & 0.21 & 0.12 & 0.04 \\
\hline
\end{tabular}

\section{A MAXIMUM ENTROPY LOW-PASS FILTER}

Rationale. The method of ME is developed as a tool for assigning a probability distribution of observing a system to be at a certain state according to information in hand, which is in the form of constraints [21]. Based on conventional studies on properties of the noise filter, Eq. (1), in the EEG source localization problems, which will be illustrated later, one can treat the weightings $p_{l}$ as a probability distribution. Thus the ME provides a robust and objective approach for determining a preferred set of $p_{l}$ once constraints relevant to noise reduction in the EEG problems are given.

The constraints. Conventional studies in EEG data analysis have shown some properties that are relevant to smooth noise using Eq. (1). First, the $p_{l}$ are positive decimal values and symmetric around $l=0$. These values obey a normalization condition $\sum_{l=-z}^{z} p_{l}=1$. Second, the preferred set of the $p_{l}$ should have noise in data being mostly removed through the $\Phi_{s}(r)$. We expand right hand side of Eq.(1) with respect to $\mathrm{r}$ according to the Taylor expansion and use $\sum_{l=-z}^{z} p_{l}=1$ and $p_{l}=p_{-l}$, which results in the odd order derivative terms in the expansion vanished. The final result is $\Phi_{s}(r)=\Phi(r)+$ $\Phi^{(2)}(r) h^{2} \delta_{2} / 2+\cdots$, where $\Phi^{(2)}(r)$ denotes exact second order derivative of clean data and so on and $\delta_{2}=\sum_{l=-z}^{z} p_{l} l^{2}$. It indicates that smoothing ability is actually related to the $\delta_{2}$ value. Summarizing the above results, suggests the weightings $p_{l}$ to be a probability distribution of having the Eq.(1) to reduce noise by the $\delta_{2}$ approximately. Thus the ME can be applied to determine the preferred set of $p_{l}$.

The preferred low-pass filter for data smoothing. The ME states that maximizing entropy, $S=-\sum_{l=-z}^{z} p_{l} \ln p_{l}$, subject to the two constraints, the normalization condition of $p_{l}$ and $\delta_{2}=\sum_{l=-z}^{z} p_{l} l^{2}$ gives a preferred form of the $p_{l}$,

$$
p_{l}=Z^{-1} \exp \left(-\alpha l^{2}\right)
$$

where $\alpha$ is a Lagrangian multiplier and partition function $Z=\sum_{l=-z}^{z} \exp \left(-\alpha l^{2}\right)$. The preferred set of $p_{l}$ is a function of $\alpha$. Namely, the ME method generates a family of $p_{l}(\alpha)$ distribution. In principle, the Lagrangian multiplier $\alpha$ can be determined by substituting Eq. (3) back into $\delta_{2}=\sum_{l=-z}^{z} p_{l} l^{2}$, which gives $\delta_{2}=-Z^{-1} \partial Z / \partial \alpha$, if the $\delta_{2}$ 
value is given. Unfortunately, the $\delta_{2}$ value is unknown at this point. Thus, we propose an alternative method to determine the $\alpha$.

Since the preferred $\alpha$ value should result in corresponding set of the $p_{l}$ to mostly reduce noise, if one can quantify noise the preferred $\alpha$ value can be determined. Although it is difficult to quantify noise, noise variance can be quantified by the autocorrelation function [3, 6]. Suppose field potentials $\Phi(r)=\Phi_{0}(r)+\varepsilon_{r}$ is repeatedly measured several times, in which a Gaussian white noise $\varepsilon_{r}$ with variance $\sigma$ is considered. For white noise, we have $\left\langle\varepsilon_{r}\right\rangle=0$. Besides, noises are uncorrelated at different data points $\mathrm{r}$ and $\mathrm{r}^{\prime},\left\langle\varepsilon_{r} \varepsilon_{r^{\prime}}\right\rangle=\sigma^{2}$ for $r=r^{\prime}$ and 0 for $r \neq r^{\prime}$ [3]. Because noise has zero mean, mean $\left\langle\Phi_{0}(r)\right\rangle$ is identical to $\Phi_{0}(r)$, noise-free data. Thus one can find autocorrelation of $\Phi(r)$ is $C_{o}=\left\langle(\Phi(r)-\langle\Phi(r)\rangle)^{2}\right\rangle=\left\langle\left(\varepsilon_{r}-\left\langle\varepsilon_{r}\right\rangle\right)^{2}\right\rangle=\sigma^{2}$, the noise variance. Similarly, one can compute the autocorrelation function of $\Phi_{s}(r), C_{\text {smooth }}=\left\langle\left(\Phi_{s}(r)-\left\langle\Phi_{s}(r)\right\rangle\right)^{2}\right\rangle=$ $\sigma^{2} \sum_{l=-z}^{z} p_{l}^{2}$. It indicates noise variance being amplified by $\sum_{l=-z}^{z} p_{l}^{2}$ after data is processed by the Eq.(1). Thus the preferred $\alpha$ value should minimize the noise variance $C_{\text {smooth }}$, which is found to be zero and $p_{l}(\alpha=0)=1 /(2 z+1)$. This is exactly the mean filter obtained from the least square method $[3,19]$. Yet the ME approach roots in reducing noise variance $C_{\text {smooth }}$ mostly, which is not clear in the least square method.

\section{FILTER OUT NOISE IN SOURCE LOCALIZATION}

Basic features. Freeman and Nicholson suggest to identify current sources and sinks through calculating second order derivative of the field potential via the $\mathrm{Ta}_{3}(r)$ with specific smoothed $\Phi_{s}(r)$. However, the $\mathrm{Ta}_{3}(r)$ does not provide accurate calculation. One straightforward way to raise the accuracy is to incorporate more data points into for calculation,

$$
\operatorname{Ta}_{2 M+1}(r)=\sum_{m=-M}^{M} a_{m}^{T} \Phi(r+m h),
$$

where $2 \mathrm{M}+1$ data points are considered. To determine coefficients $a_{m}^{T}$, one first expand $\Phi(r+m h)$ with respect to $\mathrm{r}$ based on the Taylor expansion, $\operatorname{Ta}_{2 M+1}(r)=$ $\sum_{m=-M}^{M} a_{m}^{T} \Phi(r)+\sum_{m=-M}^{M} a_{m}^{T} m h \Phi^{(1)}(r)+\sum_{m=-M}^{M} a_{m}^{T}(m h)^{2} / 2 \Phi^{(2)}(r)+\cdots$. By requesting coefficient of second order term, $\sum_{m=-M}^{M} a_{m}^{T}(m h)^{2} / 2=1$, and rests are zero, we have approximated $\mathrm{Ta}_{2 M+1}(r)$ to be identical to exact second order derivative $\Phi^{(2)}(r)$. One then can solve for coefficients $a_{m}^{T}$ given these criteria. For three point calculation, coefficients are given in the Ta 3 . Coefficients $a_{ \pm 2}^{\mathrm{T}}=-1 / 12, a_{ \pm 1}^{\mathrm{T}}=16 / 12$, and $a_{0}^{\mathrm{T}}=-30 / 12$ for $\mathrm{Ta}_{5}$ and $a_{ \pm 3}^{\mathrm{T}}=1 / 90, a_{ \pm 2}^{\mathrm{T}}=-13.5 / 90, a_{ \pm 1}^{\mathrm{T}}=135 / 90$, and $a_{0}^{\mathrm{T}}=-245 / 90$ for Ta7.

Given these coefficients, one can immediately find the noise variance to be amplified by $C_{D_{2}}=\left\langle\left(D_{2}(r)-\left\langle D_{2}(r)\right\rangle\right)^{2}\right\rangle=\sigma^{2} \sum_{m=-M}^{M}\left(a_{m}^{\mathrm{T}}\right)^{2}$ after operation of the $\operatorname{Ta}_{2 M+1}(r)$. For example, it is 6, 8.03, 9.75 times of the original noise variance for using $\mathrm{Ta}_{3}(r)$, $\mathrm{Ta}_{5}(r)$, and $\mathrm{Ta}_{7}(r)$ respectively. The noise amplification is proportional to the number of data utilized in the calculation. This result suggests a trade-off of raising the accuracy of 
TABLE 2. The preferred ME filters, denoted by $\mathrm{MES}_{\mathrm{n}} \mathrm{Ta}_{\mathrm{m}}$.

\begin{tabular}{|l|c|c|cccc|}
\hline & $C_{D_{s 2}}\left(\times \sigma^{2}\right)$ & $\alpha$ & $p_{0}$ & $p_{ \pm 1}$ & $p_{ \pm 2}$ & $p_{ \pm 3}$ \\
\hline $\mathrm{MES}_{3} \mathrm{Ta}_{3}$ & 0.28 & 0.031 & 0.42 & 0.28 & 0 & 0 \\
\hline $\mathrm{MES}_{3} \mathrm{Ta}_{5}$ & 0.38 & 0.033 & 0.43 & 0.28 & 0 & 0 \\
\hline $\mathrm{MES}_{3} \mathrm{Ta}_{7}$ & 0.42 & 0.035 & 0.44 & 0.27 & 0 & 0 \\
\hline $\mathrm{MES}_{5} \mathrm{Ta}_{3}$ & 0.048 & 0.017 & 0.29 & 0.23 & 0.12 & 0 \\
\hline $\mathrm{MES}_{5} \mathrm{Ta}_{5}$ & 0.061 & 0.018 & 0.29 & 0.23 & 0.11 & 0 \\
\hline $\mathrm{MES}_{5} \mathrm{Ta}_{7}$ & 0.065 & 0.019 & 0.30 & 0.23 & 0.11 & 0 \\
\hline $\mathrm{MES}_{7} \mathrm{Ta}_{3}$ & 0.014 & 0.011 & 0.22 & 0.19 & 0.12 & 0.06 \\
\hline $\mathrm{MES}_{7} \mathrm{Ta}_{5}$ & 0.017 & 0.012 & 0.23 & 0.2 & 0.12 & 0.05 \\
\hline $\mathrm{MES}_{7} \mathrm{Ta}_{7}$ & 0.019 & 0.012 & 0.23 & 0.2 & 0.12 & 0.05 \\
\hline
\end{tabular}

second order derivative calculation with more data is amplification of the noise variance. Thus it is unlikely that the Freeman and Nicholson's and others filters can smooth this noise amplification because those filters are designed for $\mathrm{Ta}_{3}(r)$ calculation only. One needs new smoothing functions.

The preferred ME filter. The new smoothing functions can be obtained through the following procedure. Substituting smoothed data, Eq. (1) into second order derivative $\mathrm{Ta}_{2 M+1}(r)$ first gives a generic expression

$$
D_{s 2}(r)=\sum_{n=-(M+z)}^{M+z} a_{n}^{\prime} \Phi(r+n h),
$$

where $a_{n}^{\prime}$ is a function of $a_{m}^{T}$ and $p_{l}$. The preferred set of $p_{l}$ is then determined by minimizing variance $C_{D_{s 2}}=\left\langle\left(D_{s 2}(r)-\left\langle D_{s 2}(r)\right\rangle\right)^{2}\right\rangle=\sigma^{2} \sum_{n=-(M+z)}^{M+z} a_{n}^{\prime 2}$. It should have high accuracy of second order derivative calculation and minimum noise variance simultaneously. Table 2 gives the results. We define operation based on the ME that utilizes $\mathrm{n}$ data points for noise smoothing, $S_{n}$, and $m$ data for calculating second order derivative, $\mathrm{Ta}_{\mathrm{m}}$, as $\mathrm{MES}_{\mathrm{n}} \mathrm{Ta}_{\mathrm{m}}$ in the first column of Table. 2. Second column records the minimum $C_{D_{s 2}}$ value. Next five columns list the corresponding $\alpha$ and the $p_{l}$ value consecutively. The table first shows that when there is more data, $S_{3}, S_{5}$, and $S_{7}$, to be considered for smoothing given $\mathrm{Ta}_{\mathrm{m}}$, the noise variance $C_{D_{s 2}}$ will be decreased dramatically. However, when one smoothes data with $\mathrm{S}_{\mathrm{n}}$ and calculate second order derivative using more number of smoothed data points, $\mathrm{Ta}_{3}, \mathrm{Ta}_{5}$, and $\mathrm{Ta}_{7}$, the noise variance $C_{D_{s 2}}$ will then be amplified as expected.

\section{PERFORMANCE ANALYSES}

Instead of applying the proposed ME filter directly to study real EEG data for examinations, we will only analyze the filters's performance through frequency and noise variance analysis in this work. In addition, we will compare it to other filters, mean filter (MF), Freeman and Nicholson's (FN), Rappelsberger's (R) filters from Table 1. 

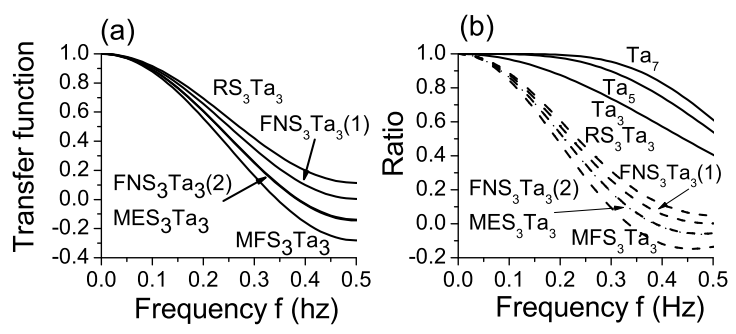

FIGURE 1. Frequency analysis of the five different 3-point $S_{3}$ filters in panel (a) and different $\mathrm{S}_{3}, \mathrm{Ta}_{3}$ in panel (b).

Frequency analysis. The frequency analysis applies the transfer function $H(\omega)$ for examining changes of signal's amplitudes after signal being processed by the filters and second order derivative calculation [3]. The transfer function $H(\omega)=$ $\exp (-i \omega r) \Phi_{s}(r)=p_{0}+2 \sum_{l=1}^{z} p_{l} \cos l \omega$ is derived by substituting $\Phi(r)=\exp (i \omega r)$, which is shown to be an eigenfunction of a linear time-invariant system, into $\Phi_{s}(r)$ of the Eq.(5), where angular frequency $\omega$ equals to $2 \pi f$ rotational frequency [3]. When the transfer function is less then one, it indicates signal amplitude being attenuated by the filter. Panel (a) of Fig. 1 plots the results of using the different filters from Table 1. This figure demonstrates the mean filter has the largest amplitude attenuation and is followed by $\mathrm{FNS}_{3}(2)$ and $\mathrm{MES}_{3}, \mathrm{FNS}_{3}(1)$, and $\mathrm{RS}_{3}$ consecutively.

Similarly, one can compute the transfer function of second order derivative of smoothed data. Afterward, we compute the ratio of calculated and real second order derivative value through $R(\omega)=-D_{s 2}(r) / \omega^{2}=-1 / \omega^{2}\left(a_{0}+2 \sum_{l=1}^{M+z} a_{l}^{\prime} \cos l \omega\right)$ for explicit comparison of effects of the smoothing. When the ratio $R(\omega)$ equals to one, the calculated second order derivative of the smoothed data is identical to real value. Panel (b) of Fig. 1 plots not only the calculation through five filters, but also the three second order derivative calculations $\mathrm{Ta}_{3}, \mathrm{Ta}_{5}$, and $\mathrm{Ta}_{7}$ respectively. First of all, frequency attenuation is the smallest in $\mathrm{Ta}_{7}$, and is followed by $\mathrm{Ta}_{5}$ and $\mathrm{Ta}_{3}$. That explains again, why the second order derivative is more accurate when more data is comprehended for calculation. Given the second order derivative calculation with fixed number of smoothed data, for example, all $\mathrm{S}_{3} \mathrm{Ta}_{3}$ in this figure, it suggests that the mean filter $\mathrm{MFS}_{3} \mathrm{Ta}_{3}$ again has signal with high frequency attenuated the most among these filters. These attenuation patterns are similar to the panel (a), which is not surprising because smoothing data first and then calculating second order derivative is identical to calculating second order derivative first and then smooth the calculation.

Noise variance analysis. It seems the mean filter to be superior to all other choices from the previous analysis. However, the following noise variance analysis shows different results. We substitute the filters from Table 1 into Eq. (4) to compute new coefficient $a_{m}^{\prime}$, and evaluate noise variance $C_{D_{s 2}}$. The results are listed in Table 3. Because we design the ME filter to mostly reduce noise variance, the analysis shows the $\mathrm{MES}_{3} \mathrm{Ta}_{3}$ filter to exactly has the best performance among all filters.

Besides, Freeman and Nicholson have found noise in field potential measurements to be proportional to a K-value, $K=\sum_{l=-(M+z)}^{M+z}\left|a_{l}^{\prime}\right|$ [2]. Thus we calculate the K-value 
TABLE 3. Comparisons of filters. The table lists $a_{n}^{\prime}$ value and noise variance $C_{D_{s 2}}\left(\times \sigma^{2}\right)$ in the third column and $K$ value derived by Freeman and Nicholson for noise estimate in the fourth column.

\begin{tabular}{|l|ccccc|c|c|}
\hline & $a_{0}^{\prime}$ & $a_{ \pm 1}^{\prime}$ & $a_{ \pm 2}^{\prime}$ & $a_{ \pm 3}^{\prime}$ & $a_{ \pm 4}^{\prime}$ & $C_{D_{s 2}}\left(\times \sigma^{2}\right)$ & $K$ \\
\hline $\mathrm{MFS}_{3} \mathrm{Ta}_{3}$ & 0 & -0.333 & 0.333 & 0 & 0 & 0.44 & 1.33 \\
\hline $\mathrm{FNS}_{3} \mathrm{Ta}_{3}(1)$ & -0.5 & 0 & 0.25 & 0 & 0 & 0.38 & 1.01 \\
\hline $\mathrm{FNS}_{3} \mathrm{Ta}_{3}(2)$ & -0.286 & 0.143 & 0.286 & 0 & 0 & 0.28 & 1.13 \\
\hline $\mathrm{RS}_{3} \mathrm{Ta}_{3}$ & -0.623 & 0.082 & 0.229 & 0 & 0 & 0.57 & 1.34 \\
\hline $\mathrm{MES}_{3} \mathrm{Ta}_{3}$ & -0.284 & -0.144 & 0.286 & 0 & 0 & 0.28 & 1.14 \\
\hline \hline $\mathrm{FNS}_{5} \mathrm{Ta}_{3}$ & -0.2 & -0.05 & 0.06 & 0.09 & 0 & 0.073 & $0.63 \mid$ \\
\hline $\mathrm{FNS}_{7} \mathrm{Ta}_{3}$ & -0.1 & -0.04 & 0.01 & 0.04 & 0.04 & 0.019 & 0.39 \\
\hline $\mathrm{MES}_{5} \mathrm{Ta}_{3}$ & -0.116 & -0.055 & -0.007 & 0.12 & 0 & 0.048 & 0.48 \\
\hline $\mathrm{MES}_{7} \mathrm{Ta}_{3}$ & -0.06 & -0.038 & 0.003 & 0.003 & -0.06 & 0.014 & 0.27 \\
\hline
\end{tabular}

after using different filters and list in the fourth column of Table 3. The K-value shows although $\mathrm{MES}_{3} \mathrm{Ta}_{3}$ is slightly outperformed by the $\mathrm{FNS}_{3} \mathrm{~T}_{3}(1)$, which has the same performance as the $\mathrm{FNS}_{3} \mathrm{~T}_{3}(2)$, the ME filter is superior than all other filters when more data points are included for smoothing.

Discussions. The frequency analysis has shown the mean filter $\mathrm{MFS}_{3}$ and the $\mathrm{MES}_{3}$ and $\mathrm{FNS}_{3}(2)$ mostly attenuate high frequency part in the signal. Yet the noise variance analysis, comparison of the $C_{D_{s 2}}$ and $\mathrm{K}$ value, shows the $\mathrm{MES}_{3} \mathrm{Ta}_{3}$ and the $\mathrm{FNS}_{3} \mathrm{Ta}_{3}(2)$ can mostly reduce noise variance among all three-point filters. These three analyses suggests the ME filter not only mostly reduce noise variance in second order derivative but also has the ability to mostly attenuate high frequency signal.

\section{CONCLUSIONS}

The filter design delicately depends on one's purpose and properties of a target system. It sometimes involves some ad hoc empirical guidelines that suit ones need. For example, Freeman and Nicholson have designed several filters for their needs [2]. We propose and demonstrate an informatic approach to design a low-pass filter based on the method of ME. In addition, we examine and compare the preferred ME filter with other well known filters in EEG studies through frequency and noise variance analysis. The performance of the preferred ME filter $\mathrm{MES}_{3} \mathrm{Ta}_{3}$ and the $\mathrm{FNS}_{3} \mathrm{Ta}_{3}(2)$ filter are found to be almost identical. Even though the ME filter is designed for second order derivative calculation especially for the EEG data in this work only, the approach's systematics and flexibility ensures one can design filters that suit other purposes robustly without introducing ad hoc empirical guidelines. 


\section{ACKNOWLEDGMENT}

This work is partially supported by NSC-95-2811-M008-016 to CYT and NSC-95-2112M-008-004 to HCL from the National Science Council, Taiwan ROC.

\section{REFERENCES}

1. J. Freeman and C. Nicholson, "Theory of current source-density analysis and determination of conductivity tensor for anuran cerebellum," J. Neurophysiol. 38, 356-368 (1975)

2. J. Freeman and C. Nicholson, "Experimental optimization of current source density technique for Anuran cerebellum," J. Neurophysiol. 38, 369-382 (1975).

3. R. Hammings, "Digital filters," 3rd Ed., London: Prentice-Hall International, Inc., 1989.

4. G. Borgiotti and L. J. Kaplan, "Superresolution of uncorrelated interference sources by using adaptive array technique," IEEE Trans. Antennas Propagat., 27, 842-845 (1979).

5. A. Restrepo, A. C. Bovik, "Adaptive trimmed mean filters for image restoration," IEEE Trans. Acous, Speech, and Signal Process., 36, 1326-1337 (1988).

6. D. D. Feldman and L. J. Grifiths, "A constrained projection approach for robust adaptive beamforming," in Proc. Int. Conf. Acoust., Speech, and Signal Processing, 1991, pp. 1357-1360.

7. K. Sekihara, S. Nagarajan, D. Poeppel, A. Marantz, and Y. Miyashita, "Reconstructing spatiotemporal activities of neural sources using an MEG vector beamformer technique," IEEE Trans. Biomed. Eng., 48, 760-771 (2001).

8. S. Sardy, P. Tseng, and A. Bruce, "Robust wavelet de-noising," IEEE Trans. Signal Process., 40, 1146-1152 (2001).

9. N. Avesta and T. Aboulnasr, "Maximum entropy Kalman filter for image reconstruction and compression," J. Electronic Imaging, 13, 738-755 (2004).

10. A. Beghdadi and A. Khellaf, "A noise-filtering method using a local information measure," IEEE Trans. Image Process., 6, 879-882 (1997).

11. G. A. Kivman, A. L. Kurapov, and A. V. Guessen, "An entropy approach to tuning weights and smoothing in the generalized inversion," J. Atmospheric and Oceanic Tech. 18, 266-276 (2000).

12. J.-W. Wu, D. Erdogmus, and J. C. Principe, "Minimizing Fisher information of the error in supervised adaptive filter training," in Proc. ICASSP, 2004, pp.513-516.

13. S. Avitente and W. J. Williams, "Minimum entropy approach to de-nosing time frequency distributions," in Proc. SPIE, 2001, vol.4474, pp. 57-67.

14. V. E. DeBrunner, L. S. DeBrunner, S. Coone, and X. Hu "Using entropy to build efficient FIT digital filters," in 2004 IEEE 11 th Digital Signal Processing Workshop and IEEE Signal Processing Education Workshop, 2004, pp. 97-101.

15. P. Rappelsberger, H. Pockberger, and H. Petsche, "Current cource density analysis: Methods and application to simultaneously recorded field potentials of the rabbit's visual cortex," Pflügers Arch., 389, 159-170 (1981).

16. U. Mitzdorf, "Current source-density method and application in cat cerebral cortex: Investigation of evoked potentials and EEG phenomena," Physiological Rev. vol.63, pp. 37-100 (1985).

17. C. J. Karwoski, X. Xu, and H. Yu, "Current-source density analysis of the electroretinogram of the frog: methodological issues and origin of components," J. Opt. Soc. Am. A, 13, 549-556 (1996).

18. I. Ulbert, E. Halgren, G. Heit, and G. Karmos, "Multiple microelectrode-recording system for human intracortical applications," J. Neuroscience Meth. 106, 69-79 (2001).

19. C. Lanczos, "Applied analysis," Englewood Cliffs., N. J.: Prentice Hall, 1956.

20. R. Hammings, "Numerical methods for science and engineers," New York: McGrow-Hill, 1971, pp. 276-322.

21. E. T. Jaynes, "Information theory and statistical mechanics," Phy. Rev. 106, 620-630 (1957). 